CERIAS Tech Report 2005-79

\title{
ACCESS CONTROL ENFORCEMENT FOR CONVERSATION-BASED WEB SERVICES
}

by M. Mecella, M.Ouzzani, F. Paci, E. Bertino

Center for Education and Research in

Information Assurance and Security,

Purdue University, West Lafayette, IN 47907-2086 


\section{Access Control Enforcement for Conversation-based Web Services}

\author{
Massimo Mecella *
}

\author{
Federica Paci ₹
}

Elisa Bertino \$

\begin{abstract}
Service Oriented Computing is emerging as the main approach to build distributed enterprise applications on the Web. The widespread use of Web services is hindered by the lack of adequate security and privacy support. In this paper, we present a novel framework for enforcing access control in conversation-based Web services. Our approach takes into account the conversational nature of Web services. This is in contrast with existing approaches to access control enforcement that assume a Web service as a set of independent operations. Furthermore, our approach achieves a tradeoff between the need to protect Web service's access control policies and the need to disclose to clients the portion of access control policies related to the conversations they are interested in. This is important to avoid situations where the client cannot progress in the conversation due to the lack of required security requirements. We introduce the concept of $k$-trustworthiness that defines the conversations for which a client can provide credentials maximizing the likelihood that it will eventually hit a final state.
\end{abstract}

\section{Categories and Subject Descriptors}

D.4.6 [Security and Protection]: [Access controls]; K.6.5 [Management of Computing and Information Systems]: Security and Protection-Authentication; H.3.5 [Information Storage and Retrieval]: Online Information Services

\section{Keywords}

Web services, Access control, Transition systems, Conversations

\footnotetext{
${ }^{*}$ Dipartimento di Informatica e Sistemistica, Università di Roma "La Sapienza", Italy; mecella@dis.uniroma1.it. This work was perfomed while visiting researcher at CERIAS and Department of Computer Sciences, Purdue University, USA. The work is also partly supported by the MIUR - FIRB 2001 Project "MAIS".

${ }^{\dagger}$ Department of Computer Sciences, Purdue University, USA; mourad@cs . purdue.edu.

${ }^{\ddagger}$ Dipartimento di Informatica e Comunicazione, Università degli Studi di Milano, Italy; paci@dico.unimi.it.

${ }^{\S}$ CERIAS and Department of Computer Sciences, Purdue University, USA; bertino@cerias.purdue.edu.
}

Copyright is held by the author/owner(s).

\section{INTRODUCTION}

Service Oriented Computing is poised to be the leading approach to build (distributed) applications on the Web using Web services as the basic building blocks. Web services provide a standard framework for interoperating independently developed Web applications. Generally speaking, a Web service is a set of related functionalities that can be programmatically accessed through the Web. These functionalities represent the different operations made available by the Web service and are described in its service description using the WSDL standard language. While many in the literature treated Web services as a set of independent single operations, interacting with real world Web services involves generally a sequence of invocations of several of their operations, referred to as conversation. A simple example is a bookstore Web service; buying a book involves generally searching for the book, browsing the details and reviews about this book, adding the book to the shopping cart, checking out, paying, etc. It is then important to represent Web services in some formalism that would represent all potential conversations that can take place between the Web service and the client.

As organizations increase their use of Web services and adopt them as the primary tool to build fairly complex distributed systems, security and policy disclosure become crucial [14]. It is well acknowledged that the wide spread adoption of Web services cannot happen without effective solutions for security issues. In this paper, we focus on access control and the limited disclosure of access control policies. An access control model restricts the set of clients or subjects that can invoke Web service's operations. Since clients ws are not known a priori, we adopt credentials to enforce access control. Credentials are signed assertions describing properties of a subject that are used to establish trust between two unknown communicating parties before allowing access to information or services. Access control policies define rules stating that only subjects with certain credentials satisfying specific conditions can invoke a given operation of the Web service.

While access control has been widely studied in the literature and especially in database systems [7], only recently work on security for Web services has emerged as an important part of the Web service saga $[19,15,1,6]$. Most approaches in the literature assume a single operation model where operations are independent from each other. Access control is either enforced at the level of the entire Web service or at the level of single operations. In the first approach, 
the Web service could ask, in advance, the client to provide all the credentials associated with all operations of that Web service. This approach guarantees that a subject will always arrive at the end of whichever conversation. However, it has the drawback that the subject will become aware of all policies on the base of which access control is enforced. Another drawback is that the client may have to submit more credentials than needed. An alternative strategy is to require only the credentials associated with the next operation that the client wants to perform. This strategy has the advantage of asking from the subject only the credentials necessary to gain access to the requested operation. However, the subject is continuously solicited to provide credentials for each transition. In addition, after several steps, the client may reach a state in which it cannot progress because the lack of credentials.

It is important to observe that Web services operations represent a coarse-grained process that takes place in the application supporting the Web service and usually involves the consumption of several resources. This shows how important it is for the Web service to maximize the chance that a user would reach a final state to avoid the risk of wasting resources. This should be balanced with the need to retain some control on the disclosure of access policies.

In this paper, we propose a conversation-based access control model that enables service providers to retain some control on the disclosure of their access control policies while giving clients some guarantees on the termination of their interactions. First, we model all possible conversations as finite transition systems (aka finite state machines) [17, 4], in which final states represent those ones in which the interaction with the client can be (but no necessarily) ended. Furthermore, our access control model attempts to to maximize the likelihood that a client reaches a final state without necessarily having to be made aware of all the access control policies. We introduce a novel concept of $k$-trustworthiness where $k$ can be seen as the level of trust that a Web service has on a client at any point of their interaction. The greater the level of trust associated with a client, the greater is the amount of information about access control policies that can be disclosed to this client. $k$ represents the length of the conversations, from the current state, such that the client is requested to provide the credentials to invoke any operation along these paths. All conversations along these paths will eventually lead to a final state after $k$ steps. Thus, the client is assured that its conversation can eventually terminate. As we shall see, based on this simple notion of $k$-trustworthiness, we are able to develop a flexible (with limited policy disclosure) access control model for conversation-based Web services.

The rest of the paper is organized as follows. Section 2 presents the related work. In Section 3, we present our conversation-based access control model for Web services. In Section 4, we introduce the algorithms used to enforce access control. In Section 5, we describe how the model can be implemented in Web service environments. Finally, in Section 6 we discuss our approach and its advantages. We also identify the major issues in extending our approach to composite services.

\section{RELATED WORK}

Recent papers $[3,4,13]$ have argued that a Web service is more than a set of independent operations. In fact, during a Web service's invocation, a client interacts with the service performing a sequence of operations in a particular order. Such a sequence is called conversation. Specifically, $[3,4]$ adopt a model based on finite transition systems (aka finite state machines) for representing all possible conversations. The approach of [13] is based on the combined use of two Web service languages, WS-Conversation (WSCL) and WS-Agreement, that allows one to specify non-trivial conversations in which several messages have to be exchanged before the service is completed and/or the conversation may evolve in different ways depending on the state and the needs of the requesting agents and of the service provider.

As far as security issues in Web services, a fair amount of related research in this area is due to the industry, with Regarding security issues in Web services, two major standards have emerged Security Assertion Markup Language (SAML) and eXtensible Access Control Markup Language (XACML). SAML defines an XML framework for exchanging authentication and authorization information for securing Web services. XACML is an XML framework for specifying access control policies for web-based resources. Recently it has been extended to specify access control policies for Web services. Other emerging specifications include WS-Security and WS-Policy. WS-Security is a specification for securing SOAP messages using XML Encryption and XML Signature standards and attaching security credentials thereto. WS-Policy is used to describe the security policies in terms of their characteristics and supported features (such as required security tokens, encryption algorithms, privacy rules, etc.).

These proposals do not address the issue of enforcing access control policies. Several approaches $[19,15,1,6]$ suggest some preliminary ideas, but none of them provide a comprehensive solution. [19] proposes two RBAC (Role Based Access Control) models, SWS-RBAC, for single Web services, and CWS-RBAC, for composite Web services. In both models, a service has a few access modes and a role is associated with a list of services which clients, who are assigned that role, have permission to execute. In CWSRBAC model, the role to which a client is assigned to access a composite service, must be a global role, which is mapped onto local roles of the service providers of the component Web services. [15] proposes an approach for specifying and enforcing security policies. These are specified using a language called WebGuard based on temporal logic and are processed by an enforcement engine to yield site and platform-specific access control. This code is integrated with a Web server and platform specific libraries to enforce the specified policies on a given Web service. [1] presents a Web service architecture for enforcing access control policies expressed in WS-Policy. The architecture is similar to the one proposed in the XACML standard and is characterized by three main components: PDP (Policy Decision Point), PEP (Policy Enforcement Point) and PAP (Policy Administration Point). The PDP realizes the interface between a service and the access control architecture. When a client requests to invoke a service, the service forwards the request to the PDP, which, in turn, sends it to the PEP. The PEP 
asks to the PAP for the policies applicable to the request and evaluates it against the applicable policies. Then, it returns the final decision to the PDP, which issues the service access.

[6] present WS-AC1, an access control model with flexible granularity in protecting objects and negotiation capabilities. WS-AC1 is based on the specification of policies stating conditions on the values of the identity attributes and of the service parameters that a client must provide to invoke the service. Conditions may also be specified against context parameters, such as time. Further, it is possible to define fine-grained policies by associating them with a specific service as well as coarse-grained policies, to be applied to a class of services. The negotiation capabilities of WS-AC1 are related both to identity attributes and to service parameters. Through a negotiation process, the client is driven toward the specification of an access request compliant with the service description and policies.

The work in [16] considers the issue of modeling and managing trust policies and how to assure that an enforcement system can migrate, during negotiation of trust policies, from a previous and no-more valid set of policies to a new one. Trust policies are modeled as finite state machines, and lifecycle management is obtained by managing such formalizations. This work is indeed orthogonal and complementary to ours, even if the basic formalism is the same: our focus is on enforcing access control during service execution, whereas their focus is on modeling and managing trust negotiations. Clearly the two approaches can be merged, e.g., our the trust policies can be managed according to their model.

All these proposals describe policy-driven access control models. They are based on the enforcement of access control policies stating the requirements to be satisfied by a client to be granted access to a Web service. Since a Web service can be invoked potentially by anyone, the requirements are expressed as conditions on the digital credentials owned by a client. But all these models assume that a Web service provides just a single operation, or that all operations are independent.

\section{CONVERSATION-BASED ACCESS CONTROL}

In the proposed model, a Web service is characterized by the set of operations that it exports and constraints on the possible conversations it can execute. We compactly represent the service conversations as a finite transition system.

Definition 1. (Transition System). Let $\mathcal{W S}$ be a Web service. The transition system of $\mathcal{W S}$ is a tuple $\mathcal{T} \mathcal{S}=$ $\left(\Sigma, S, s_{0}, \delta, F\right)$ where $\Sigma$ is the alphabet of operations offered by the service, $S$ is a finite set of states, $s_{0} \in S$ is the single initial state, $\delta: \subseteq S \times \Sigma \rightarrow S$ is the transition function, and $F \subseteq S$ is the set of final states(states in which a conversation may end, but does not necessarily have to).

If $\delta\left(s_{i}, a\right)=s_{j}$, we represent this as $s_{i} \stackrel{a}{\longrightarrow} s_{j}$, and we call $a$ the label of the transition. The transition function can be extended to finite length sequences of operations or conversations (traces [17]). Given a conversation $c: a_{1}, a_{2}, \ldots a_{n}$, and two states $s_{0}$ and $s_{n}$, we say that $s_{0}$ evolves in $s_{n}$ (rep-

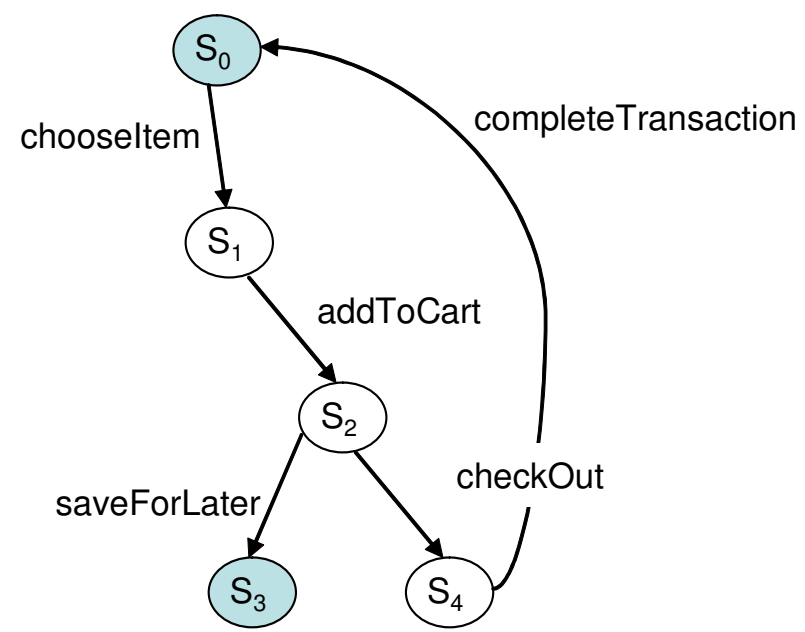

Figure 1: eShop service's transition system

resented as $\left.s_{0} \stackrel{c}{\Longrightarrow} s_{n}\right)$ iff $\exists s^{\prime}$ such that $s_{0} \stackrel{a_{1}}{\longrightarrow} s^{\prime}$ and $s^{\prime} \stackrel{a_{2}, \ldots a_{n}}{\Longrightarrow} s_{n}$.

EXAMPLE 1. Figure 1 represents the transition system of a simple retail Web service eShop, selling some goods. The different labels represent the operations that a client can invoke from any given state and are self-explaining. Final states are represented by gray circles. A client can be involved in different conversations with the service. The client can choose for an item (chooseItem), then can add to the cart (addToCart). Further, the client can decide to buy the item (checkOut and then completeTransaction operations) or postpone the purchase (saveForLater) and end the interaction.

Credentials are the mean to establish trust between a client and the service provider. They are assertions about a given client, referred to as the owner, issued by trusted third parties called Certification Authorities (CAs). They are digitally signed using the private key of the issuer CA and can be verified using the issuer's public key. A credential contains typically a set of arbitrary properties characterizing the owner and are specified via (name,value) pairs. Each credential has a type based on the set of attribute names in the credential.

Definition 2. (Credential). A credential $\mathcal{C}$ is a tuple (Issuer, Owner, $\mathcal{T}$ ype, Attr) where $\mathcal{I}$ ssuer is the name of the $C A$ who issues the credential, Owner is the name of the credential owner, $\mathcal{T}$ ype identifies the type of the credential. and $\mathcal{A t t r}=\left(\mathcal{A}_{i}, \ldots, \mathcal{A}_{n}\right)$ is the set of attributes characterizing the $\mathcal{T}$ ype of the credential. An attribute $\mathcal{A}_{i}$ is a pair (name $_{\mathcal{A}_{i}}$, value $\left.\mathcal{A}_{i}\right)$, where name $\mathcal{A}_{i}$ is the name of the attribute $\mathcal{A}_{i}$ and value $_{\mathcal{A}_{i}}$ is a value in the attribute domain $\operatorname{dom}_{\mathcal{A}_{i}}$ of $\mathcal{A}_{i}$.

Conditions on the attributes in a credential specify the security requirements of the service provider. An attribute condition $\mathcal{A C}$ is an expression of the form name A $_{i}$ op $k$, where name $_{\mathcal{A}_{i}}$ is an attribute name, op is a comparison operator and $k$ is a constant value in $\operatorname{dom}_{\mathcal{A}_{i}}$. We say that a 
credential $\mathcal{C}:(\mathcal{I}$ ssuer, $\mathcal{O}$ wner, $\mathcal{T}$ ype, $\mathcal{A}$ ttr $)$ satisfies an attribute condition $\mathcal{A C}$ : name $_{\mathcal{A}_{i}}$ op $k$ (denoted as $\mathcal{C} \triangleright \mathcal{A C}$ ) if and only if $\exists \mathcal{A}_{l} \in \mathcal{A}$ ttr such that name $_{\mathcal{A}_{l}}=$ name $_{\mathcal{A}_{i}}$ in $\mathcal{A C}$ and value $\mathcal{A}_{l}$ makes true name $\mathcal{A}_{i}$ op $k$.

We denote with term $\mathcal{T}$ a couple $\langle\mathcal{T}$ ype $\mathcal{C}, \mathcal{C C}\rangle$, where $\mathcal{T}$ ype $\mathcal{C}$ is a credential type and $\mathcal{C C}$ is a set (eventually empty) of attribute conditions. These attribute conditions are combined using classical boolean operators.

Definition 3. (Operation Access Control Policy). Let $\mathcal{T S}=\left(\Sigma, S, s_{0}, \delta, F\right)$ be the transition system of a Web service $\mathcal{W S}$ and o the identifier of an operation in $\Sigma$. An operation access control policy for $o$ is an expression of the form $\mathcal{P}: o \leftarrow \mathcal{T}_{1}, \mathcal{T}_{2}, \ldots \mathcal{T}_{n}, n \geq 1$ where $\mathcal{T}_{1}, \mathcal{T}_{2}, \ldots \mathcal{T}_{n}$ are terms and $o$ is the Web service operation guarded by the access control policy.

The semantics of an access control policy is that, given a set of credentials, the access to the operation is granted iff the credentials satisfies all the attribute conditions (i.e., $\mathcal{C} \triangleright \mathcal{T}_{1}$ and $\mathcal{C} \triangleright \mathcal{T}_{2}$ and $\left.\ldots \mathcal{C} \triangleright \mathcal{T}_{n}\right)$. As discussed in many logical formalizations (e.g., $[9,11])$, the access to the Web service operation can be checked through a reasoning service that verifies if the access request is a logical consequence of the policy and the credentials.

Definition 4. (Conversation Access Control Policy). Let $\mathcal{T} \mathcal{S}=\left(\Sigma, S, s_{0}, \delta, F\right)$ be the transition system of a Web service $\mathcal{W S}, c$ : $a_{1}, \ldots a_{k}$ a conversation in $\mathcal{S}, a_{1}, \ldots a_{k}$ identifiers of operations in $\Sigma$, and $\mathcal{P}_{1}$ : $a_{1} \leftarrow \mathcal{T}_{11}, \ldots \mathcal{T}_{1 n^{1}}, \ldots, \mathcal{P}_{k}: a_{k} \leftarrow \mathcal{T}_{k 1}, \ldots \mathcal{T}_{k n^{k}}$ the corresponding operation access control policies. A conversation access control policy for $c$ is an expression of the form: $c \leftarrow \mathcal{T}_{11}, \ldots \mathcal{T}_{1 n^{1}}, \ldots \mathcal{T}_{k 1}, \ldots, \mathcal{T}_{k n^{k}}$ where $\mathcal{T}_{11}, \ldots \mathcal{T}_{1 n^{1}}, \ldots \mathcal{T}_{k 1}$ is the conjunction of the terms in $\mathcal{P}_{1} \ldots \mathcal{P}_{k}$.

This definition captures the intuition that a client, owing a set of credentials satisfying a conversation access control policy is granted access to all the operations constituting the conversation. If the conversation is such that it reaches a final state, then the satisfaction of the policy assures that the client will be authorized up to reaching its own goal. The service provider will not be forced to deny access to some operations in the middle of the conversation due to lack of authorization.

Example 1 (cont.) An example of access control policies for operations addToCart and saveForLater are respectively:

$\mathcal{P}_{1}:$ addToCart $\leftarrow$ CreditCard_Holder $($ Type $=$ MasterCard $)$

$\mathcal{P}_{2}:$ saveForLater $\leftarrow$ Subscribed_Member.

Policy $\mathcal{P}_{1}$ states that only the clients having a MasterCard can perform operation addToCart, while policy $\mathcal{P}_{2}$ authorizes only the subscribed clients to execute saveForLater. The conversation access control policy for the conversation $C$ : addToCart $\cdot$ saveForLater is:

$C \leftarrow$ CreditCard_Holder $($ Type $=$ MasterCard $)$,

Subscribed_Member.

DEFINITION 5. (Trustworthiness Level). Let $\mathcal{T S}=$ $\left(\Sigma, S, s_{0}, \delta, F\right)$ be the transition system of a Web service $\mathcal{W S}$ and $s \in \mathcal{S}$ be a state. A trustworthiness level for $s$ is the length of a conversation such that $s \stackrel{c}{\Longrightarrow} t$ ) with $t \in F$.
A trustworthiness level represents the length of a conversation, from a given state $s$ in the transition system, that leads to a final state.

Definition 6. (k-Trust Policy). Let $\mathcal{T S}=$ $\left(\Sigma, S, s_{0}, \delta, F\right)$ be the transition system of Web service $\mathcal{W S}$ and $k$ a trustworthiness level computed on $s \in \mathcal{S}$. $A$ $\mathrm{k}$-trust policy is an expression of the form $k_{s} \leftarrow \mathcal{T}_{1}, \mathcal{T}_{2}, ., \mathcal{T}_{n}$, $n \geq 1$, where $\mathcal{T}_{1}, \mathcal{T}_{2}, \ldots \mathcal{T}_{n}$ are terms and $k_{s}$ is a trustworthiness level for state $s$.

A k-trust policy states the type of credentials and the conditions on the credential attributes a client, in state $s$, must provide in order to be assigned to a trustworthiness level $k$. We use the concept of trustworthiness level to limit the disclosure of service provider's access control policies. Therefore, when a client is assigned the trustworthiness level $k$ (on the basis of an appropriate k-trust policy), the enforcement system asks only the credentials needed to satisfy all the conversation access control policies associated with the conversations from the current state to final states and having length less or equal to $\mathrm{k}$.

Example 1 (cont.) Let us consider the start state (labeled with $S_{0}$ ) in Figure 1 and determine the potential conversations. These potential conversations that lead to a final state and that we need to consider to compute the k-trustworthiness levels are: (1) chooseItem · addToCart · saveForLater; chooseItem.addToCart.checkOut.completeTransaction; and (3) chooseItem.addToCart.checkOut. completeTransaction.chooseItem.addToCart. saveForLater. Adding more conversations will be useless from access control perspective since the same conversation will be repeated. Hence there are 3 different k-trustworthiness levels: $\{3,4,7\}$. For instance, the $k_{3}$-trust policy to assign a client to trustworthiness level 3 is of the form $k_{3} \leftarrow$ PictureID $($ Age $>18)$ : it means that if the client is older then eighteen is entrusted with trustworthiness level 3. Such a client has to fulfill the access control policies associated with the conversations having length less or equal to 3. These conversations include the following operations: \{chooseItem\}, addToCart, and saveForLater\}.

\section{ACCESS CONTROL ENFORCEMENT}

The main feature of the enforcement system proposed in this paper is that access control is enforced by considering conversations, thus maximizing the likelihood that a client reaches a final state and does not drop off due to lack of authorization. The idea is to determine, whenever needed and in any step of the interaction with the client (i.e., at each state of the transition system), the appropriate trustworthiness level $\mathrm{k}$ to assign to the client. This requires knowing all potential trust levels at any state. The level assigned to the client is determined based on the k-trust policies. If the client provides the required credentials, the likelihood that it will end up to a final state without lacking authorizations is high. Clearly, the client is not forced to follow one of the conversations it has been authorized to; this is why we refer to "high likelihood" and not to "guarantee" that the client will reach a final state. At some states of the conversations 
defined by $\mathrm{k}$, the client may decide to take a different conversation that is not included in the ones it has been authorized to, a longer conversation for example. If this is the case, the current trustworthiness level $k$ is recalculated on the basis of the potential levels at the current state and new credentials are required.

The challenge now is that given a transition system, we need to determine for each state all possible trustworthiness levels from that state as well as the possible conversations that would define the corresponding access policies. We base our solution on the following observations:

- For an acyclic transition system, the set of potential paths leading from any state to any final state is finite. This set can be easily calculated by a simple traversal of the graph of the transition system.

- If from a given state, a conversation involves a cycle, an infinite number of paths are possible to arrive to a final state within or while traversing this cycle.

- Since we are dealing with access control policies, usually if an access control policy of an operation $a$ has been checked against a client, we do not have to check it again if the client invoke the operation $a$ more than once.

We clearly see that the main difficulties in traversing the transition system and determining the potential conversations relate to the existence of cycles. Before going further, let us introduce the concept of strongly connected component (SCC for short). A strongly connected component is the maximal subgraph of a directed graph such that for every pair of vertices $u, v$ in the subgraph, there is a directed path from $u$ to $v$ and a directed path from $v$ to $u$ [18]. The transition system of a Web service can be regarded as a directed graph where a transition between two states is a directed edge, without considering the labeling. Based on the above concept, an acyclic graph can be produced where nodes represent the different strongly connected components of the initial graph. This graph is called the directed graph of the strongly connected components and is noted $\mathcal{G}^{s c c}$. It can be efficiently computed trough the classical Tarjan's algorithm [18] or more recent optimizations, e.g., [12].

We can then make the following observations on this new graph: (i) if the initial $\mathcal{T S}$ is acyclic then $\mathcal{G}^{s c c}$ is $\mathcal{T S}$ itself [10]; (ii) the nodes that are not involved in cycles will remain unchanged in the new graph; (iii) cycles will be "collapsed" into strongly connected components and need to be dealt with in a appropriate way.

For any SCC, we need to determine all possible conversations that will lead from an in-going node, i.e., coming from outside the component, to an out-going node, i.e., going outside the component. These conversations should have the properties to cover all potential operations within that strongly connected component. The overall idea of the algorithm which finds all potential k-trustworthiness levels for all states, will be: for a given state, determine all subsequent strongly connected components, including the one to which the current state belongs to. The algorithm will then traverse the transition system from that state and record all conversations leading to a final state. By having computed all possible conversations of all strongly connected components, we will be able to find finite conversations even in the case of cycles.

Before giving the details of the algorithm, we will need to introduce several concepts.

An object type SetOf<element $\rangle^{1}$ for representing set of <element>s, where <element> can be whichever other object type. We use the term set in a proper way, to mean a collection of elements without repetitions and without any order. We assume to have the = operator on sets, which compares two homogeneous sets for equality, a method add (<element> e) for adding a new element, and || for calculating its dimension (number of elements). An object type Sequence is defined for representing conversations. On such a type, some methods are defined: (i) length ( ) for having the length of the sequence, and (ii) set ()$\rightarrow$ Set0fOperation, which returns the set of all the distinct operations. As an example, $a c g \cdot \operatorname{set}()=\{a, c, g\}=a c g c g c g \cdot \operatorname{set}()$.

The directed graph of the strongly connected components of the original transition system is defined as follows:

DEFINITION 7. (Graph of $\mathrm{SCC})$. Given a transition system $\mathcal{T} \mathcal{S}=\left(\Sigma, S, s_{0}, \delta, F\right)$, the directed graph of the strongly connected components $\mathcal{G}^{s c c}=\left\langle S^{s c c}, E^{s c c}\right\rangle$ is the graph with nodes $N^{s c c}$ and oriented edges $E^{s c c}$ obtained as follows:

$N^{s c c}=\{c: \quad c$ is a strongly connected component in $\mathcal{T} \mathcal{S}\}$; $E^{s c c}=\left\{\left\langle c_{1}, c_{2}\right\rangle: c_{1} \neq c_{2}\right.$ and $\exists a \in \delta, s_{1} \in c_{1}, s_{2} \in c_{2}$ such that $\left.s_{1} \stackrel{a}{\longrightarrow} s_{2}\right\}$

Given a state $s \in \mathcal{T S}$, we refer to the node of $G^{s c c}$ associated to the strongly connected component of $s$ as $c(s)$; we also say that $c(s)$ is the image of $s$.

In the algorithms presented in the following, we assume an object type GraphSCC, defined for representing a graph of sCC. On the type GraphSCC, a method projection (Node c) $\rightarrow$ GraphSCC is defined, that takes as input a node $c$ and returns a new subgraph obtained by considering $c$ and all nodes reachable by it, i.e., it is the subgraph obtained by visiting depth-first the graph starting from $c$.

For each node/sCC of $\mathcal{G}^{s c c}$, we know the number of different operations that label transitions among the associated states of $\mathcal{T S}$. More specifically details, for each $\mathrm{SCC} c$, the set $\mathcal{O}_{c}=\left\{a\right.$ such that $s_{1} \stackrel{a}{\longrightarrow} s_{2}$ and $\left.c\left(s_{1}\right)=c\left(s_{2}\right)=c\right\}$ can be easily determined. The cardinality of $\mathcal{O}_{c}$ is referred to as $\operatorname{card}(c)$. If the connected component $c$ is the image of a single state of $\mathcal{T S}$, then its $\operatorname{card}(c)=0$. On the type GraphSCC, we define a method card(Node c) $\rightarrow$ Integer that takes as input a node $c$ and returns its cardinality.

For each node/sCC of $\mathcal{G}^{s c c}$, the longest path, among the shortest ones that (i) starting from an in-going node finish in a distinct out-going node, and (ii) comprise all the different operations in $\mathcal{O}_{c}$. More specifically, for each SCC $c$, a sequence of operations str is said to be covering iff $e \stackrel{\text { str }}{\Longrightarrow} o$ with $e$ in-going state of $c$ and $o$ out-going state $(c(e)=c(o)=c)$ AND str.set ()$=\mathcal{O}_{c}$. We use the notation $\widetilde{s t r}$ for referring to a traversing sequence of operations 2. Then for each $\operatorname{SCC} c$, a set $\mathcal{C}_{c}$ is defined: $\mathcal{C}_{c}=\left\{\widetilde{s t r_{i}}\right.$

${ }^{1}$ In the following, we use Type for indicating object types, and object for indicating object instances. Moreover we will sloppy on many obvious object types.

${ }^{2} \mathrm{~A}$ traversing sequence is not necessarily an Eulerian path 
such that: $\left(o_{i} \neq o_{j}\right.$ for $\left.i \neq j\right)$ and $\left(\forall s t r_{k}\right.$ with $o_{i}=o_{k}$ then $s r_{k}$. length ()$\geq s t r_{i}$. length( $\}$. We denote coverage (c) $=\max \left(s t r_{i} \in C_{c}\right)$.

The coverage of a SCC can be calculated by generating by enumeration, which can be done with a simple recursion, all the paths from any in-going node to an out-going one. A global array of boolean variables, with dimension equal to the number of distinct out-going nodes, is used to record whether the out-going node has been reached, and another global array of integer variables maintains the length of the sequence that evolves the in-going node up to the out-going one. As soon as all the boolean variables are set to true, meaning that we have found the shortest paths, then the maximum among the values in the other array is calculated. In order to close all the recursive instances, each of them is controlled by a condition on the conjunction of all the boolean array's values.

On the type GraphSCC, a method coverage (Node c) $\rightarrow$ Integer is defined, that takes as input a node $c$ and returns its coverage.

For each node/sCC of $\mathcal{G}^{s c c}$, we define the rank as follows:

$$
\operatorname{rank}(c)= \begin{cases}\operatorname{coverage}(\mathrm{c}) & \\ 1+\operatorname{coverage}(c) & \text { if } \mathrm{c} \text { is the root of } \mathcal{G}^{s c c} \\ +\max (\operatorname{rank}(m)) & \\ & \text { where } m \text { are all the } \\ & \text { possible predecessors } \\ & \text { of } c\end{cases}
$$

As $\mathcal{G}^{s c c}$ is acyclic, the rank of each node can be computed in three steps: (i) by running a depth-first-search algorithm, and for each visited node to push on a stack a record, labeled with the node, containing the predecessor node; (ii) then by popping the stack, and for each encountered record, to remove from the stack all the records with the same state, by recording the corresponding predecessor node, and to push in another stack the formula for calculating the rank (at this point the predecessors are all correctly identified); (iii) finally, by popping the second stack, and for each removed record, we calculate the rank. We should observe that the stack now gives the exact order according to which to calculate the formulas: each removed record gives the values to be used in following records.

Finally, on the type GraphSCC, a method rank (Node c) $\rightarrow$ Integer is defined, that takes as input a node $c$ and returns its rank.

We can now present the algorithms for computing, for each state of the Web service transition system, the possible $\mathrm{k}$-levels and the conversations corresponding to them. These algorithms assume some global variables, on which all instances of the recursion have shared access. These variables are the transition system $\mathcal{T S}$ of type TS, the $\mathcal{A C}-$ Set of type SetOfPolicy that, for each operation, report the corresponding access control policy $\mathcal{P}$ (of type Policy), the $\mathcal{G}^{s c c}$ (of type GraphSCC) and a $C-B a g$ (of type SetOfSequence), which is built during the execution of the algorithms, and represents the set of conversations defining the k-levels.

of $c$, whereas each Eulerian path, if exists, is a traversing sequence. This is why we do not impose the uniqueness of an edge.

\section{Algorithm 1: isNewString()}

Input: $b$ : Boolean

Output:

$$
\begin{array}{lc}
(1) & \text { foreach } x \in C-B a g \\
(2) & \text { if } x . \operatorname{set}()=\text { this.set }() \\
(3) & \text { return (false); } \\
(4) & \text { return (true); }
\end{array}
$$

The isNewString( () is a method defined on the object type Sequence.

Algorithm 2: build()

Input: $s$ : State, str: Sequence

Output:

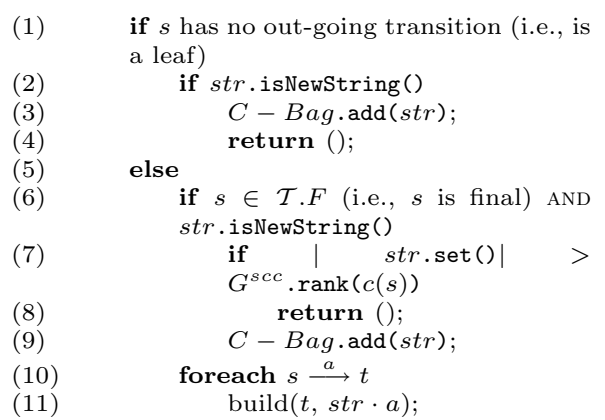$$
\text { if } s \in \mathcal{T} . F \text { (i.e., } s \text { is final) AND }
$$

Algorithm 3: buildBag\&KLevels()

Input: $s$ : State

Output: $C-B$ ag: Set0fSequence,

$K-B a g:$ SetOfInteger

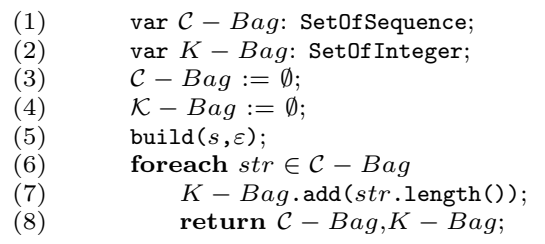

The overall algorithm builds, for each state of the Web service transition system, the k-levels and the corresponding conversations.

Algorithm 4: computeOverallBag\&KLevels()

(1) $\quad \operatorname{var} \mathcal{C}-$ Bag - Set: SetOfSetOfSequence;

(2) var $K-B a g-$ Set: SetOfSetOfInteger;

(3) foreach $s \in \mathcal{T} . \Sigma$

(4) $\quad\{\mathcal{C}-\operatorname{Bag}[s], K-\operatorname{Bag}[s]\}:=$ buildBag\&KLevels $(s)$; 


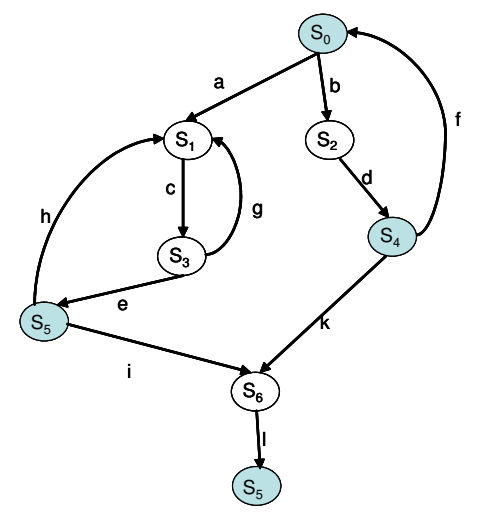

(a) (b)

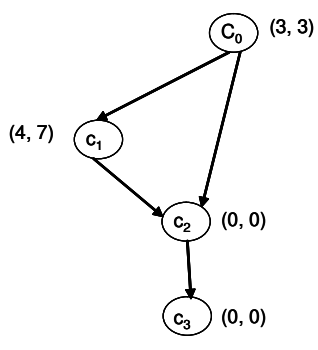

Figure 2: A transition system (a) with its $\mathcal{G}^{s c c}$ (b)

EXAMPLE 2. Figure 2(a) represents the transition system of some Web service. This transition system can be reduced to the graph in figure 2(b) containing four strongly connected components. The different states, representing each a strongly connected component, are labeled with pairs $(x, y)$ representing the maximum number of symbols (operations) and the coverage of that strongly connected component respectively. These numbers are then used to calculate all the $k$-trustworthiness levels of all states in the transition system. For example, the k-trustworthiness levels assigned to $S_{1}$ are $\{2,4,5,7,9\}$. The longest conversation being $\mathrm{c} \cdot \mathrm{g} \cdot \mathrm{c} \cdot \mathrm{e} \cdot \mathrm{h} \cdot \mathrm{c} \cdot \mathrm{e} \cdot \mathrm{i} \cdot \mathrm{l}$.

Now that for each state we computed all potential k-trustworthiness levels and corresponding conversations (from which to derive conversation policies), the access control enforcement system can proceed through the following phases:

- Bootstrapping phase - This phase occurs when the subject has its first contact with the Web service. The enforcement system assigns the initial level of trust $k$, amongst all possible ones (as computed previously), based on the set of initial credentials provided by the client (e.g., the IP address of the client) and the trust policies of the Web service. If the initial credentials are not sufficient, the access control system assign to him the smallest level of trust, or a default level of 1 (meaning step-by-step access control), or refuse the access, depending on the trust policies.

- Once the access control enforcement system assigns a level of trust $\mathrm{k}$ to the client, it will ask the client to provide all the required credentials based on the associated access policies computed by the previous algorithm.

- If the subject provides the requested credentials, it can invoke all operations on paths less or equal to $\mathrm{k}$ that lead to final states.

- If from a given state, the client decides to continue its interaction with the Web service through a path different from those assigned by its k-trustworthiness level, then a new k-level of trust needs to be computed and assigned to the client. The process will then continue as before until the client decides to stop.

\section{ARCHITECTURE OF THE ENFORCE- MENT SYSTEM}

This section describes how the proposed access control model for conversation-based Web services can be implemented in Web service environments. The system architecture is depicted in Figure 3. To be compliant with the XACML standard, the access control enforcement system is composed of a Policy Enforcement Point (PEP), a Policy Decision Point (PDP) and a Policy Administration Point (PAP). The PEP realizes the interface with clients and with the Execution Controller System (ECS) [2]. The ECS is not part of the enforcement system: it maintains a copy of the transition system to keep track of the state of the conversation between the client and the service. Further, at deployment time, it generates a table reporting for each state the $k$-trustworthiness levels.

The PEP intercepts all the access requests submitted by clients, specifying the name of an operation the client wants to perform and/or a set of credentials.

The first request that a client sends to the PEP contains both a name of an operation and a set of credentials (step 1). Once received, the PEP contacts the ECS to provide it information about the operation requested, so it can update the state of the conversation and can return it to the PEP with the table (steps 2-3). Then, the PEP, reformulates the access request adding information about the current state of the conversation and the table and sends it to the PDP (step 4). The PDP's k-Trustworthiness Level Assignment Module (TLA), having received from the PEP the information about the current state of the conversation and the table, queries the table to select the trustworthiness levels $k_{1}, \ldots, k_{n}$. Hence, the TLA Module interacts with the PAP which manages the policies, to retrieve the k-trust policies associated with trustworthiness levels $k_{1}, \ldots, k_{n}$ (step 5-6) and evaluates if the credentials provided by the client in the request satisfy one of the policies. If this is the case, the client is assigned to the trustworthiness level $k_{i}$ associated with the $k_{i}$-trust policy he is compliant with. Once assigned the trustworthiness level $k_{i}$, the TLA sends it with the associated conversations to the PDP's Policy Selection Module (PS)(step 7). The PS asks the PAP for the access control policies related to the operations constituting the conversations, that the client may engage with the service on the basis of the assigned trustworthiness level $k_{i}$ (steps 8-9). Then, the PS module combines the selected policies to obtain the corresponding conversation access control policy. Hence, it returns the policies to the PEP with $k_{i}$ (step 10). At this point, the PEP asks to the client to provide the credentials required by the policies and evaluates them against the policies (steps 11-12). If the check is positive, the client can perform any operation in the conversations related to the trustworthiness level $k_{i}$. Since the PEP stores a copy of the table of trustworthiness levels and the level $k_{i}$ assigned to the client, when it submits a request to perform an operation, which does not belong to the allowed conversations, the PEP contacts the PDP, which assigns a new trustwor- 


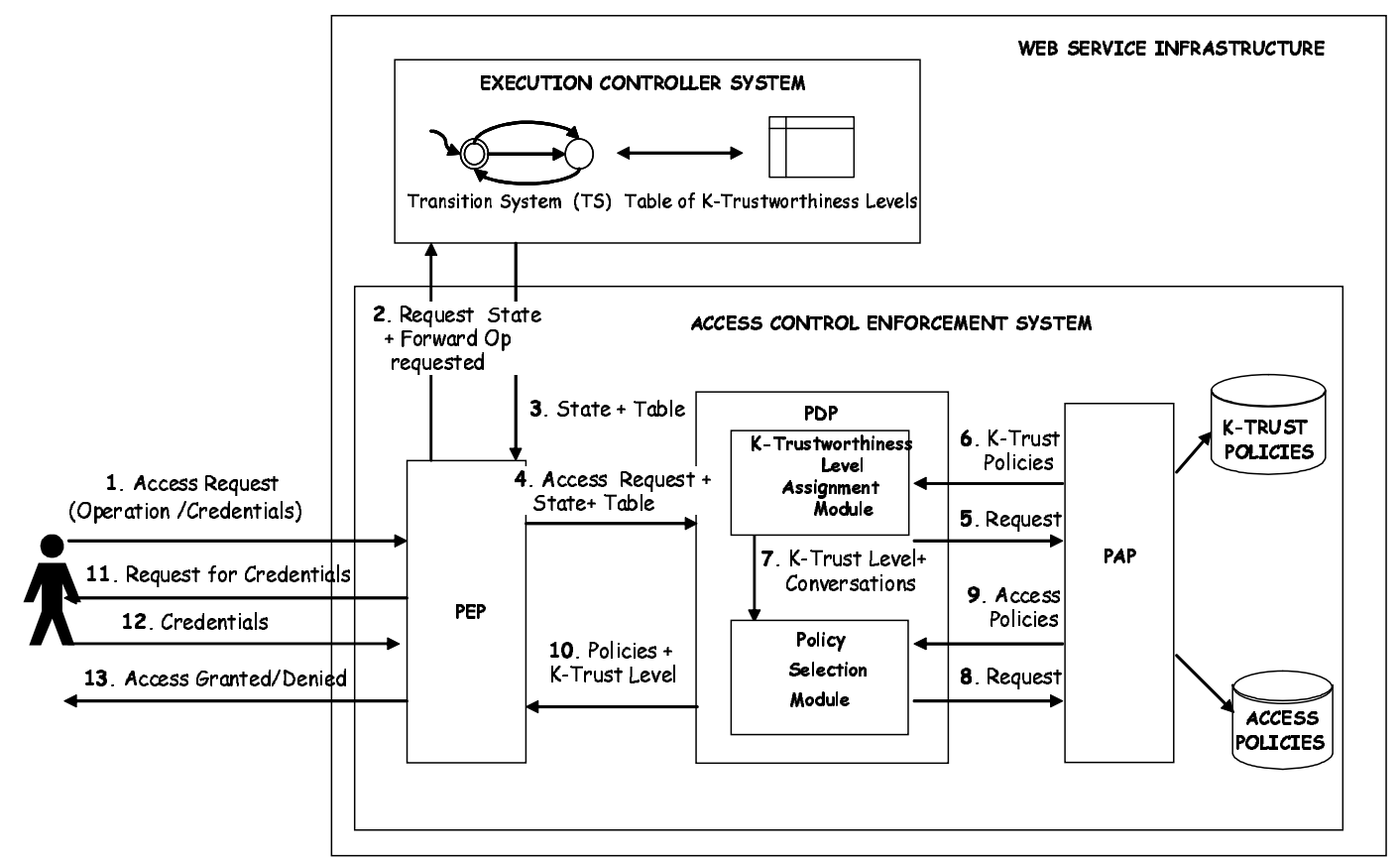

Figure 3: System Architecture

thiness level to the client. In this case, the PEP does not send again the table of trustworthiness levels, but only the state of the conversation with the client, which is necessary to select from the table the trustworthiness levels associated with that state.

The main advantage of the proposed architecture is that is in conformity with the reference standard for access control in distributed systems, XACML. Further, it is modular and allows an easy integration of the access control system in Web service frameworks. Finally, the enforcement system is independent from the language used to express k-trust and access control policies. Both WS-Policy and the XACML Profile for Web services can be used to represent the policies characterizing our model.

\section{DISCUSSION AND FUTURE WORK}

In this paper, we presented a novel approach to deal with access control in conversation-based Web services. Our first contribution is to consider Web services as transition systems instead of systems that present sets of independent operations. As we mentioned earlier, most existing access control approaches assume a single operation model for Web service where the invocation of operations are independent from each other.

Moreover, as mentioned previously, two extreme approaches regarding the disclosure of access policies are possible: (1) requesting all the credentials needed for all the operations, and (2) requesting credentials related to each operation the client is interested in invoking. In this context, our second objective was to strike a balance between the need to reveal only part of the Web service access policy and the need to offer enough assurance to clients that they can reach a final state.
In order to support such claims, we develop a very simple model for measuring the two parameters risk and disclosure. Given a Web service operation $a$, we consider $\mathcal{P}_{a}$ as the probability that the client DOES NOT have the credential(s) satisfying the access control policy guarding the operation.

In general, the risk associated to an event is the product of the probability that event happens and the damage produced by the event. In simple terms, the damage of having the client dropping off due to lack of authorization is the number of executed operations. Indeed, executing an operation requires resources to the service provider, and if the conversation is suddenly interrupted in a non-final state, all these resources have been "wasted". In addition, the leakage in terms of disclosure of access control policies is proportional to the operations already executed. We now evaluate these two simple metrics risk and leakage faced by a service provider during a conversation conv $=\left(a_{1}, \ldots, a_{n}\right)$.

In a step-by-step enforcement, the risk faced before involving the $i$-th operation ( $a_{i}$ being the next operation for which the client may not posses the required credential to access to) is:

$$
\mathcal{R}_{i}=\mathcal{P}_{a_{i}} \cdot(i-1) \quad i=1 \ldots n
$$

Similarly, the leakage after executing the $i$-th operation invocation $\left(a_{i}+1\right.$ being the next operation) is:

$$
\mathcal{L}_{i}=\mathcal{P}_{a_{i+1}} \cdot i \quad i=1 \ldots n
$$

In this case, the client may be an attacker that voluntarily drop-off the conversation after accessing the previous policies.

In our conversation-based enforcement, assuming that the conversation conv is the one for which the service provider 


\begin{tabular}{|l|l|l|}
\hline Metric & Step-by-step & Conversation \\
\hline Risk $: \sum_{i=1}^{n} \mathcal{R}_{i}$ & $\mathcal{P} \frac{n \cdot(n-1)}{2}$ & 0 \\
\hline Leakage $: \mathcal{L}_{n}$ & $n$ & $n$ \\
\hline
\end{tabular}

Table 1: Risk and Leakage Evaluation

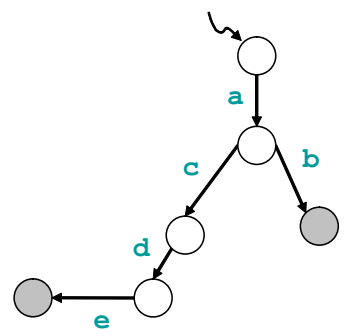

Figure 4: A simple transition system

has requested all the credentials, we have:

$$
\mathcal{R}_{i}=\Pi_{i=1}^{n} \mathcal{P}_{a_{i}} \cdot 0=0 \quad i=1 \ldots n
$$

Indeed during the conversation, the various invocations of Web service operations are somehow independent. Thus, the probability that a client has all the credential(s) needed to access all the operations is simply $\prod_{i=1}^{n} \mathcal{P}_{a_{i}}=\mathcal{P}_{a_{1}} \cdot \ldots \cdot \mathcal{P}_{a_{n}}$. However, the service provider has requested credentials for this conversation, the damage is always 0 . The system is safe about the client having the requirements to reach the end of the conversation. This is not necessary since the client can choose a different operation.

Since the enforcement system has required all the credentials at the beginning, the leakage is:

$$
\mathcal{L}_{i}=\mathcal{P}_{a_{i}} \cdot n \quad i=1 \ldots n
$$

Table 1 summarizes the risk and leakage, after the conversation conv, for our approach and for a step-by-step approach assuming that all $\mathcal{P}_{a_{i}}$ are equals, i.e, $\forall i: \mathcal{P}_{a_{i}}=\mathcal{P}$.

Let us now compare the possible approaches to access control enforcement on a simple Web service having the behavior represented as in Figure 4.

Table 2 shows the results for the step-by-step enforcement, the conversation-based (with the 2 possible k-levels, 2 and 4), and the request-all approach. We consider both the possible conversations. Specifically, in the case of the conversation acde, with the client assigned a k-level of 2 , if after one step the client chooses an operation it has not been authorized yet, and it is assigned a k-level of 4 - the only possible, the risk is given by the damage - 1 step - for $3 \cdot \mathcal{P}$ (the probability of not having the credential for the remaining 3 steps).

Hence, it becomes clear that the k-level model is a tradeoff between the request-all approach, that always minimizes the risk by maximizing the disclosure, and the step-by-step, which minimizes the disclosure by maximizing the risk. If good client profiles (obtained by logs, etc.) are available, the trust policies can be fine tuned to have most of the clients assigned to the correct k-level, i.e., the one that effectively the client will follow, thus obtaining the best of the two extreme approaches.

Table 3 summarizes the advantages and disadvantages of the different approaches regarding access control and disclo-

\begin{tabular}{|l|l|l|l|l|}
\hline Metric & $\begin{array}{l}\text { step-by- } \\
\text { step }\end{array}$ & $\begin{array}{l}\text { k-level: } \\
\mathbf{2}\end{array}$ & $\begin{array}{l}\text { k-level: } \\
\mathbf{4}\end{array}$ & $\begin{array}{l}\text { request- } \\
\text { all }\end{array}$ \\
\hline \hline ab & \multicolumn{5}{|l|}{} \\
\hline \hline Risk & $2 \cdot \mathcal{P}$ & 0 & 0 & 0 \\
\hline Leakage & 2 & 2 & 5 & 5 \\
\hline \hline acde & \multicolumn{5}{|l}{} \\
\hline \hline Risk & $6 \cdot \mathcal{P}$ & $0+3 \cdot \mathcal{P}$ & 0 & 0 \\
\hline Leakage & 4 & 5 & 5 & 5 \\
\hline \hline
\end{tabular}

Table 2: Comparison of the various approaches on a simple Web service

sure of access policies. It shows that our solution takes a more balanced approach and provides more flexibility. On one hand, it gives some guarantees to the client that once it provides the requested credentials, it will eventually reach a final state. On the other hand, the Web service retain some control on the disclosure of its access policy.

\begin{tabular}{|l|l|l|}
\hline $\begin{array}{l}\text { Policy Dis- } \\
\text { closure }\end{array}$ & Advantages & Disadvantages \\
\hline $\begin{array}{l}\text { Disclose the } \\
\text { entire access } \\
\text { policy }\end{array}$ & $\begin{array}{l}\text { The client can reach } \\
\text { any final state if } \\
\text { it possesses the re- } \\
\text { quired credentials }\end{array}$ & $\begin{array}{l}\text { The client has access } \\
\text { to the entire policy }\end{array}$ \\
\hline $\begin{array}{l}\text { Disclose only } \\
\text { the portion as- } \\
\text { sociated with } \\
\text { the requested } \\
\text { operation }\end{array}$ & $\begin{array}{l}\text { The client has very } \\
\text { on the access policy }\end{array}$ & $\begin{array}{l}\text { The client is so- } \\
\text { and mated frequently } \\
\text { state in which it } \\
\text { cannot progress }\end{array}$ \\
\hline $\begin{array}{l}\text { Disclose only } \\
\text { sociated with } \\
\text { k-trust level }\end{array}$ & $\begin{array}{l}\text { Only a small por- } \\
\text { tion of the policy is } \\
\text { disclosed. It maxi- } \\
\text { mizes the likelihood } \\
\text { the client reaches a } \\
\text { final state. }\end{array}$ & $\begin{array}{l}\text { The client may still } \\
\text { take a path different } \\
\text { from the authorized } \\
\text { ones }\end{array}$ \\
\hline
\end{tabular}

Table 3: Access control strategies

As part of our future work we would like to integrate our approach with an exception-based mechanism tailored to support access control enforcement. In particular, in a step-by-step approach, whenever a client cannot complete a conversation because of the lack of authorization, some alternative actions and operations are taken by the Web service. A typical action would be to suspend the execution of the conversation, ask the user to acquire the missing credentials, and then resume the execution of the conversation; such a process would require investigating a number of issues, such as determining the state information that need to be maintained, and whether revalidation of previous authorizations is needed when resuming the execution. A different action would be to determine whether alternative operations can be performed to replace the operation that the user cannot execute because of the missing authorization. We would like to develop a language according to which one can express the proper handling of error situations arising from the lack of authorization.

A natural next step for our work is to extend our access control model to composite services. We need composition when a client request cannot be satisfied by any available service, but by suitably combining parts of available Web services. Composition involves usually two different issues [5]: synthesis is concerned with synthesizing a specification of how to coordinate the component services to fulfill the client request; orchestration relates to the enactment of 
the composite service and the coordination among services, by executing the specification produced by the composition synthesis.

Access control needs to be addressed at the orchestration level to manage the client's credentials needed to access the different service components and the access policies of these services. The objective is to extend the notions of conversation access control policy and $k$-trustworthiness to composite services. We are still assuming a conversation-based model for Web services. In addition, all component services support our $k$-trustworthiness model. Thus each service compute a local $k$ or trust level and the challenge would be to compute a global trust level in an effective and efficient way. We need to determine the credentials to request from the client that will lead to a final state.

In addition, Web services may fail or ask for credentials that cannot be provided by clients. In addition to the ideas presented earlier on suspending the current conversation or replacing the operations, another potential approach is to to devise a substitutability scheme [8] where the "failing" service is substituted with a new Web service that has at least the same behavior and that is access control-compatible with the composite service and the current state. This would require addressing several challenging issues including computing a new global trust level, deriving new credentials from existing ones, and devising techniques on how to deal with the work done so far by the Web service being substituted.

\section{REFERENCES}

[1] C. Ardagna, E. Damiani, S. De Capitani di Vimercati, and P. Samarati. A web service architecture for enforcing access control policies. In Proceedings of 1 st International Workshop on Views on Designing Complex Architectures, 2004.

[2] B. Benatallah, F. Casati, H. Skogsrud, and F. Toumani. Abstracting and enforcing web service protocol. International Journal of Cooperative Information Systems, 13(4), 2004.

[3] B. Benatallah, F. Casati, and F. Toumani. Web service conversation modeling: A cornerstone for e-business automation. IEEE Internet Computing, 8(1):46 - 54, 2004.

[4] D. Berardi, D. Calvanese, G. De Giacomo, M. Lenzerini, and M. Mecella. Automatic service composition based on behavioral descriptions. International Journal of Cooperative Information Systems, 14(4):333 - 376, 2005.

[5] D. Berardi, D. Calvanese, G. De Giacomo, R. Hull, and M. Mecella. Automatic Composition of Transition-based Semantic Web Services with Messaging. In Proc. VLDB 2005.

[6] E. Bertino, L. Martino, F. Paci, and A. Squicciarini. An adaptive access control model for web services. To appear on International Journal of Web Services Research (JWSR), 2005.

[7] E. Bertino and R. Sandhu. Database Security Concepts, Approaches, and Challenges. IEEE Transaction on Dependable and Secure Computing, 2(1), January-March 2005.
[8] Lucas Bordeaux, Gwen Salaün, Daniela Berardi, and Massimo Mecella. When are two web services compatible? In Technologies for E-Services, 5th International Workshop, TES 2004, Toronto, Canada, August 2004.

[9] S. De Capitani di Vimercati and P. Samarati. Access control: Policies, models and mechanisms. In R. Focardi and F. Guerrieri, editors, Foundations of Security Analysis and Design - Tutorial Lectures, volume 2171 of $L N C S$. Springer Verlag, 2001.

[10] A. Dovier, C. Piazza, and A. Policriti. An efficient algorithm for computing bisimulation equivalence. Theoretical Computer Science, 311(1-3):221-256, 2004.

[11] H. Koshutanski and F. Massacci. Interactive credential negotiation for stateful business processes. In Proc. iTrust 2005, 2005.

[12] E. Nuutila and E. Soisalon-Soininen. On finding the strongly connected components in a directed graph. Information Processing Letters, 49:9-14, 1993. An extended version as: E. Nuutila and E. Soisalon-Soininen, On Finding the Strong Components in a Directed Graph, Helsinki University of Technology, Laboratory of Information Processing Science, Technical Report TKO-B94, 1993.

[13] S. Paurobally and N. R. Jennings. Protocol engineering for web services conversations. Engineering Applications of Artificial Intelligence, 18, 2005.

[14] Kent E. Seamons, M. Winslett, and T. Yu. Limiting the Disclosure of Access Control Policies during Automated Trust Negotiation. In Proceedings of the Network and Distributed System Security Symposium, San Diego, California, USA, 2001.

[15] E.Gn Sirer and K. Wang. An access control language for web services. In Proc. ACM SACMAT 2002.

[16] H. Skogsrud, B. Benatallah, and F. Casati. Trust-serv: Model-driven lifecycle management of trust negotiation policies for web services. In Proc. $W W W$ 2004 .

[17] C. Stirling. Modal and temporal logics for processes. In F. Moller and G.M. Birtwistle, editors, Logics for Concurrency. Structure versus Automata (8th Banff Higher Order Workshop, 1995, Proceedings), volume 1043 of LNCS. Springer Verlag, 1996.

[18] R.E. Tarjan. Depth-first search and linear graph algorithms. SIAM Journal on Computing, 1:146-160, 1972.

[19] R. Wonohoesodo and Z. Tari. A role based access control forweb services. In Proc. SCC 2004, 2004. 